\title{
UNSTRUCTURED LEVEL-SET METHOD FOR SATURATED LIQUID-VAPOR PHASE CHANGE
}

\author{
N. Balcázar-Arciniega ${ }^{1, *}$, J. Rigola $^{1}$ and A. Oliva ${ }^{1}$ \\ ${ }^{1}$ Universitat Politécnica de Catalunya (UPC), Heat and Mass Transfer Technological Center (CTTC) - \\ Heat Engines Department, 08222 Terrassa (Barcelona) Spain, nestor.balcazar@upc.edu, \\ http://www.cttc.upc.edu/
}

Key words: Conservative Level-Set Method, Finite-Volume Method, Unstructured Meshes, VaporLiquid Phase Change, Flux-Limiters

\begin{abstract}
A novel conservative level-set method for saturated liquid-vapor phase change on unstructured meshes is introduced. Transport equations are discretized by the finite-volume method on collocated unstructured grids. Mass transfer promoted by thermal phase change is computed using the energy jump condition at the interface, as a function of the temperature gradient. The fractional-step projection method is used for solving the pressure-velocity coupling, convective terms are discretized by unstructured flux-limiter schemes, central difference scheme is used for discretization of diffusive terms. Verification and validation cases have been undertaken to prove the accuracy and robustness of the numerical methods, including simulation of the Stefan problem, and film boiling on a cylindrical surface. Excellent agreement between numerical solutions against analytical solution and empirical correlations from the literature is reported.
\end{abstract}

\section{INTRODUCTION}

The liquid-vapor phase change denotes a broad range of phenomena in nature and industry. Multiple examples arise from the chemical processing industry, petrochemical refineries, and conventional or nuclear thermal power plants. Boiling is a necessary step in evaporators, distillation towers, or steam generators. Evaporation of droplets is used in cooling towers to reduce water temperature or combustion of droplets of liquid fuels. Despite its technological importance, the liquid-vapor phase change is not well understood yet, whereas their predictive models are far from satisfactory.

There are three ways in which such phenomena can be explored: i) experimentally, through laboratoryscale measurements with the appropriate instrumentation, ii) using theoretical methods to get analytical solutions of simplified mathematical models, and iii) through numerical and computational methods. The complexity of multiphase flows limits theoretical methods to problems with particular conditions [1]. Furthermore, experimental measurements can be challenging to perform with sufficient control to obtain reproducible results or constrained by optical access. Thus, the development of computational methods is well justified, as it is often the only mechanism to unravel the complexities of liquid-vapor phase change.

Concerning the computational approach for multiphase flows, three methods are remarked: Euler-Euler method (E-E) such as the two-fluid model [40], Euler-Lagrange method (E-L) [40] and Direct Numerical 
Simulation (DNS) [40, 60]. In the E-E method, continuous and dispersed phases are fully interpenetrating continua. The E-E method structure is well developed; however, closure relationships between fluid phases are necessary. In the E-L method, the continuous fluid phase is formulated in the Eulerian framework. In contrast, the position and velocity of bubbles or droplets (dispersed phase) are tracked following the Lagrangian approach. In the E-L method, the closing relationships for the interaction forces between the phases must be given. On the other hand, DNS of the Navier-Stokes equations, where all scales of fluid motion are solved together with detailed interface resolution, has emerged as a powerful tool for researching multiphase flows due to the growing power of supercomputers.

Numerous methods have been reported for DNS of two-phase flows, which can be categorized based on the underlying scheme used for the advection of the fluid interface, for instance: Volume of Fluid (VoF) [29, 43, 60], level-set (LS) [38, 53, 24], coupled VoF-LS [52, 51, 12], conservative level-set (CLS) $[37,10,15]$, and front-tracking (FT) [57]. In further steps, some of the aforementioned interface capturing/tracking methods have been extended to deal with liquid-vapor phase change phenomena. For instance, $[63,28,64,62,39,34]$ have reported VoF based implementations. Extensions of LS methods have been reported in [47, 50, 48, 49, 44]. [23, 35, 54] combine LS method and ghost-fluid approach [25] to incorporate jump conditions in two-phase flow with phase change. Further efforts in the framework of coupled VoF-LS methods were reported in $[36,56,33]$. The FT method has been extended to computations of phase change by $[31,22,21,59,30,41]$. Although previous efforts have reported remarkable numerical and physical findings, most of these methods have been implemented on structured and cartesian meshes. As a consequence, many other configurations and flow conditions of industrial interest are not explored yet. Mo unstructured CLS method this research aims to intro the unstructured CLS noth deal with film-boiling on This research is organized as follows: Section 2

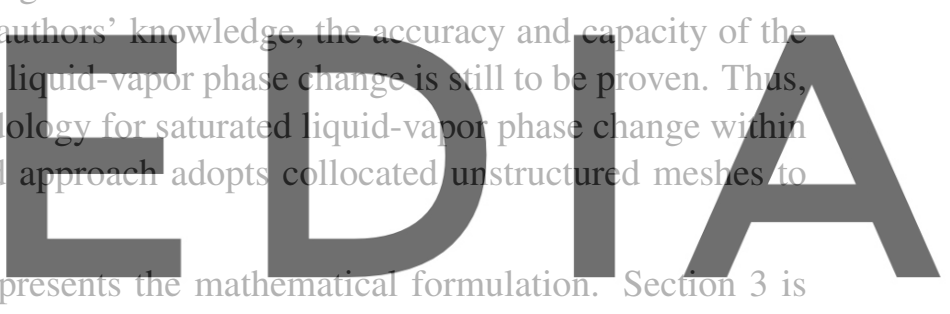
focused on the numerical methodology and its implementation on unstructured meshes. Verifications,

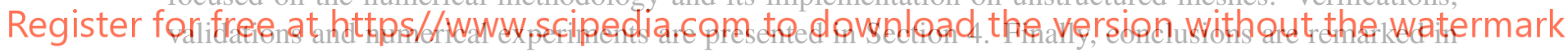
Section 5.

\section{MATHEMATICAL FORMULATION}

\subsection{Incompressible two-phase flow}

The Navier-Stokes equations for the vapor phase $\left(\Omega_{v}\right)$ and liquid phase $\left(\Omega_{l}\right)$ are introduced in the framework of the so-called one fluid formulation $[58,40,61]$ in $\Omega=\Omega_{v} \cup \Omega_{l}$ :

$$
\frac{\partial}{\partial t}(\rho \mathbf{v})+\nabla \cdot(\rho \mathbf{v v})=-\nabla p+\nabla \cdot \mu\left(\nabla \mathbf{v}+(\nabla \mathbf{v})^{T}\right)+\rho \mathbf{g}+\mathbf{f}_{\sigma},
$$

where $\mathbf{v}$ denotes the fluid velocity, $p$ is the pressure field, $\rho$ is the fluid density, $\mu$ is the dynamic viscosity, $\mathbf{g}$ is the gravitational acceleration, $\mathbf{f}_{\sigma}$ denotes the surface tension force per unit volume concentrated at the interface $(\Gamma)$. Physical properties are assumed to be constant at each fluid-phase with a jump discontinuity at $\Gamma$ :

$$
\rho=\rho_{l} H_{l}+\rho_{v} H_{v}, \mu=\mu_{l} H_{l}+\mu_{v} H_{v},
$$


where subscripts $v$ and $l$ denote the vapor phase and liquid phase respectively, $H_{v}$ is the Heaviside step function that is one in $\Omega_{v}$ and zero elsewhere, and $H_{l}=1-H_{v}$. At the discretized level, physical properties are regularized across $\Gamma$ to avoid numerical instabilities (Section 2.2).

If $\dot{m}_{l v}$ denotes the surface mass transfer rate promoted by the liquid-vapor phase change, then mass conservation equation for the vapor phase and liquid phase are written as follows:

$$
\frac{\partial}{\partial t} H_{v}+\nabla \cdot\left(H_{v} \mathbf{v}\right)=\frac{\dot{m}_{l v}}{\rho_{v}} \delta_{\Gamma}, \frac{\partial}{\partial t} H_{l}+\nabla \cdot\left(H_{l} \mathbf{v}\right)=-\frac{\dot{m}_{l v}}{\rho_{l}} \delta_{\Gamma},
$$

where $\delta_{\Gamma}$ is the Dirac delta function concentrated at $\Gamma$. The sum of previous equations lead to:

$$
\nabla \cdot \mathbf{v}=\left(\frac{1}{\rho_{v}}-\frac{1}{\rho_{l}}\right) \dot{m}_{l v} \delta_{\Gamma}
$$

which indicates that the flow is incompressible in $\Omega$, except at the interface $(\Gamma)$.

\subsection{Interface capturing: Unstructured conservative level-set method}

The unstructured conservative level-set (CLS) method proposed in Balcázar et al.[10][15] is here extended for interface capturing with phase change. In CLS methods the interface $(\Gamma)$ is implicitly represented by the 0.5 iso-surface of a regularized indicator function, $\phi(\mathbf{x}, t)=0.5(\tanh (d(\mathbf{x}, t) /(2 \varepsilon))+1)$,

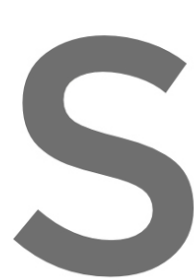
where $d(\mathbf{x}, t)$ is the signe

face profile. At the cell $\Omega$ stated. The CLS function as follows: $H_{l}^{s}=1-H^{s}$ Eq.(3),
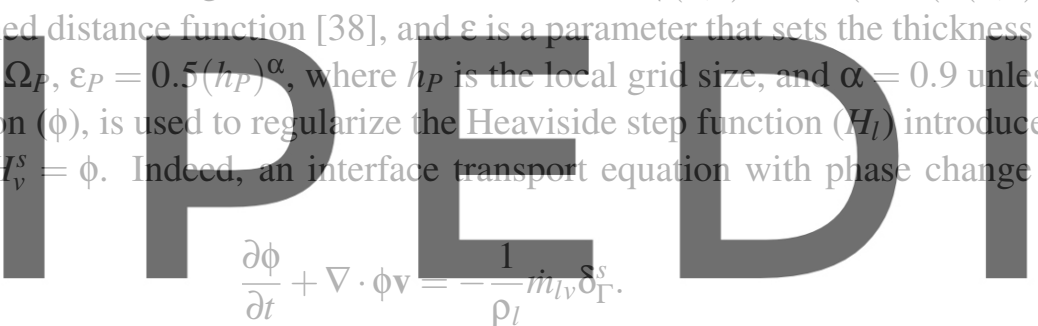

$\rho_{l}$

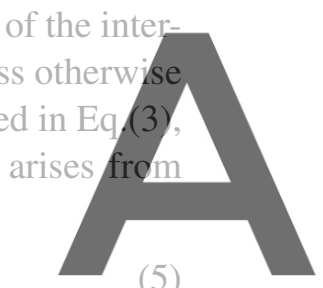

(5)

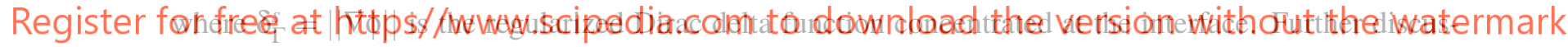
sions on the regularization of $H_{l}^{s}$ and $\delta_{\Gamma}^{s}$, in the framework of the CLS method, can be found in [15]. Furthermore, a re-initialization equation is introduced to keep a sharp and constant CLS profile [10],

$$
\frac{\partial \phi}{\partial \tau}+\left.\nabla \cdot \phi(1-\phi) \mathbf{n}\right|_{\tau=0}=\nabla \cdot \varepsilon \nabla \phi
$$

Eq.(6) is advanced in pseudo-time $\tau$ up to the steady-state; $\left.\mathbf{n}\right|_{\tau=0}$ denotes the normal unit vector, perpendicular to the interface, evaluated at $\tau=0$. Geometrical information at the interface, which includes normal unit vector (n) and curvature ( $\kappa)$, are evaluated as $\mathbf{n}(\phi)=\nabla \phi_{i}\left\|\nabla \phi_{i}\right\|^{-1}$ and $\kappa(\phi)=-\nabla \cdot \mathbf{n}$. Surface tension force is introduced in the framework of the Continuous Surface Force (CSF) model [18]: $\mathbf{f}_{\sigma}=\sigma \kappa \mathbf{n} \delta_{\Gamma}^{s}=\sigma \kappa(\phi) \mathbf{n}(\phi)\|\nabla \phi\|=\sigma \kappa(\phi) \nabla \phi$. Here, $\sigma$ is the surface tension coefficient. Finally, fluid properties in Eq. (2) are smoothed by using the regularized Heaviside step function, $\rho=\rho_{l} H_{l}^{s}+\rho_{v} H_{v}^{s}, \mu=$ $\mu_{l} H_{l}^{s}+\mu_{v} H_{v}^{s}$, where $H_{l}^{s}=\phi$ and $H_{v}^{s}=1-H_{l}^{s}$, consistently with Eq.(5). 


\subsection{Energy equation and temperature gradient driven phase change}

In this work, the energy transport equation is solved for the unsaturated vapor phase $\left(\Omega_{v}\right)$,

$$
\frac{\partial T}{\partial t}+\nabla \cdot(\mathbf{v} T)=\frac{1}{\rho c_{p}} \nabla \cdot(\lambda \nabla T)
$$

with thermal conductivity $\lambda=\lambda_{v}$, and specific heat capacity at constant pressure $c_{p}=c_{p, v}$. The temperature of the vapor-liquid interface is equal to the saturation temperature at the considered pressure, $T_{s a t}$, which is a common approximation in numerical simulations of liquid-vapor phase change [46]. On the other hand, temperature at the saturated liquid phase is $T(\mathbf{x}, t)=T_{\text {sat }}$. Mass transfer promoted by the liquid-vapor phase change $\left(m_{l v}\right)$ is obtained from an energy balance at the interface:

$$
\dot{m}_{l v}=\frac{-\lambda_{l}(\nabla T \cdot \mathbf{n})_{l}+\lambda_{v}(\nabla T \cdot \mathbf{n})_{v}}{h_{l v}}, \text { in } \Gamma,
$$

where $h_{l v}$ is the heat of vaporization. Since $T=T_{\text {sat }}$ in $\Omega_{l}$, then $(\nabla T \cdot \mathbf{m})_{l}=0$. In addition $(\nabla T \cdot \mathbf{n})_{v}=\Theta_{n, v}$ is extended on $\phi_{\text {cut }}<\phi<1$, where $\phi_{\text {cut }}=0.3$, solving the equation: $\frac{\partial}{\partial \tau^{\prime}} \Theta_{n, v}+\mathbf{n} \cdot \nabla \Theta_{n, v}=0$, which is advanced for the pseudo-time $\tau^{\prime}$.

\section{NUMERICAL METHODS}

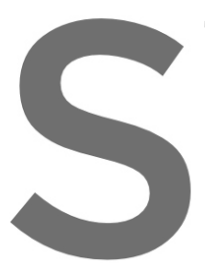

Transport equations are

15]. The convective term

(Eq.(5)), and energy equatid

(Superbee) schemes propo

$[10,15]$, whereas a linear
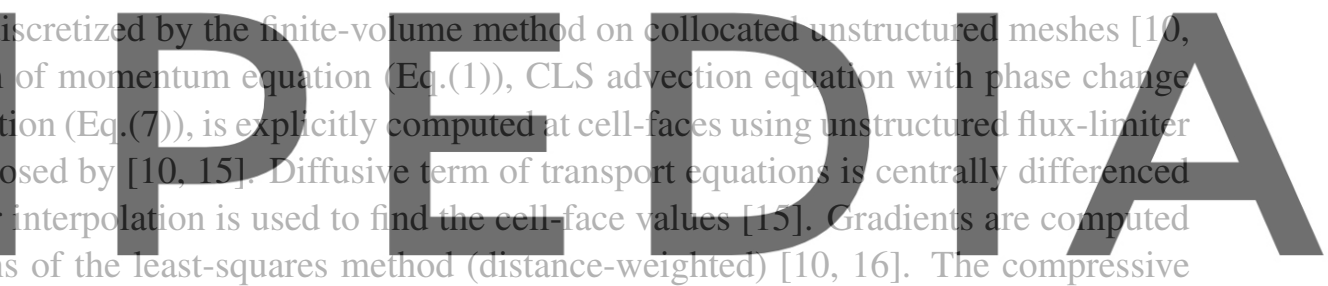

term, $\phi(1-\phi)$, in Eq.(6), is computed at cell-faces by linear interpolation [15],

Register for free at https //www.scipedia.com to download the version without the watermark

The resolution of the pressure-velocity coupling is performed by the fractional-step projection method

$[20,40,60]$. Indeed, a predictor velocity $\left(\mathbf{v}^{*}\right)$ is computed at cell-centroids:

$$
\frac{\rho \mathbf{v}^{*}-\rho^{0} \mathbf{v}^{0}}{\Delta t}=\mathbf{C}_{\mathbf{v}}^{0}+\mathbf{D}_{\mathbf{v}}^{0}+\rho \mathbf{g}+\sigma \kappa \nabla_{h} \phi
$$

Here, super-index 0 denotes the previous time step, $\mathbf{D}_{\mathbf{v}}=\nabla_{h} \cdot \mu \nabla_{h} \mathbf{v}+\nabla_{h} \cdot \mu\left(\nabla_{h} \mathbf{v}\right)^{T}, \mathbf{C}_{\mathbf{v}}=-\nabla_{h} \cdot(\rho \mathbf{v v})$. Taking the divergence of the corrector-step (Eq.(11)), and imposing the incompressibility constraint with phase change (Eq.(4)), a Poisson equation for the pressure field at cell-centroids is obtained,

$$
\nabla \cdot \frac{\Delta t}{\rho} \nabla p=\nabla \cdot \mathbf{v}^{*}-\left(\frac{1}{\rho_{v}}-\frac{1}{\rho_{l}}\right) \dot{m}_{l v} \delta_{\Gamma}^{s},\left.\mathbf{e}_{\partial \Omega} \cdot \nabla p\right|_{\partial \Omega}=0,
$$

which is computed by using a preconditioned conjugate gradient method [32]. In a further step, a corrected velocity ( $\mathbf{v})$ is computed at cell-centroids:

$$
\frac{\rho \mathbf{v}-\rho \mathbf{v}^{*}}{\Delta t}=-\nabla_{h} p
$$


In order to avoid the pressure-velocity decoupling on collocated meshes [42], a cell-face velocity $\mathbf{v}_{f}$ $[10,14]$ is interpolated, and employed to advect momentum (Eq.(1)), CLS function (Eq.(5)), and temperature (Eq.(7)) [14]. The global algorithm consists of the following steps: (1) Initialization of variables. Computation of (2) Time-step. (3) Mass transfer by phase change, Eq.(8).(4) Interface advection and reinitialization, Eq.(5) and Eq.(6). (5) Update of physical properties, and interface curvatures. (6) Energy equation, Eq.(7). (7) Fractional-step projection method, Eq.(9), Eq.(10) and Eq.(11). (8) Interpolation of velocity at cell-faces, in terms of volume-flux, mass-flux or normal velocity. (9) Repeat steps $\mathbf{2 - 8}$.

The reader is referred to [15] for additional technical details on the finite-volume discretization of transport equations on collocated unstructured meshes. The novel numerical methods for liquid-vapor phase change have been implemented in the framework of the CFD platform TermoFluids [55], which employs $\mathrm{C}++$ for object oriented design, and MPI (Message Passing Interface) for parallel communications. The parallel scalability (strong speedup) of the unstructured multiphase solver has been reported for thousands of CPU-cores in $[9,15]$.

\section{NUMERICAL EXPERIMENTS}

Validations, verifications and extensions of the unstructured CLS method without phase change [10, 15] have been reported in our previous works, for instance: buoyancy-driven rising bubbles [10, 11, 9, 5, 4], thermocapillary-driven motion of droplets $[14,6]$, bubbly flows $[13,9,15,16]$, falling droplets [8], binary droplet collision with bouncing outcome [13], bouncing collision of a droplet against a fluid-fluid

interface [13], Taylor bubbles [26,27,4], gas-liquid jets [45], do formation of droplets under shear stresses
$[2,12]$, non-Newtonian two-phase ffow [3], and mass transfer in bulbbly flows [1], 16, 7]. Furthermofe,
a comparison of the unstructured CLS method [10] and coupled VolF-LS method [12] is reported in [8].
Therefore, this research is a further step to develop an unstruetured CLS solver with phase change.
4.1. Stefan problem
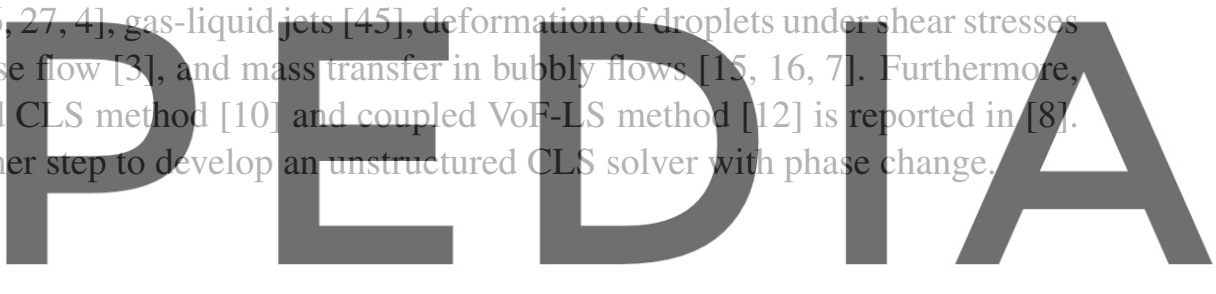

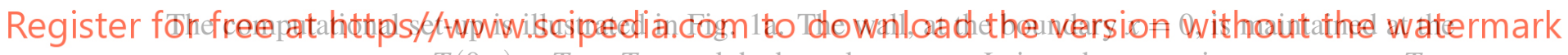
constant temperature $T(0, t)=T_{w}>T_{\text {sat }}$, and the boundary at $x=L_{x}$ is at the saturation temperature $T_{\text {sat }}$. As liquid-vapor phase change takes place, the interface located at $x_{\Gamma}(t)$ is displaced from $x=0$ to $x=L_{x}$, whereas the temperature field $T(x, t)$ varies within the vapor phase. On the other hand, temperature in the liquid phase is maintained at $T_{\text {sat }}$.

The problem parameters are summarized as follows: $T_{w}=1, T_{s a t}=0, h_{l v}=2, \sigma=24.5,\left\{\rho_{l}, \mu_{l}, \lambda_{l}, c_{p, l}\right\}=$ $\{1000,10,0.1,1\},\left\{\rho_{v}, \mu_{v}, \lambda_{v}, c_{p, v}\right\}=\{100,1,0.1,1\}, \mathbf{g}=\mathbf{0}$. The computational domain is replicated as $1 \mathrm{D}$, using 20 cells in $y$-axis and 1 cell in $z$-axis, whereas the domain length in the $x$-axis is $L_{x}=1$. Three grid sizes are selected $h=\{1 / 400,1 / 800,1 / 1600\}$. The problem is initialized with an interface at $x_{\Gamma, 0}=h$, using the analytical solution for the temperature field [1] .

The analytical solution for $T(x, t)$ and $x_{\Gamma}(t)$ has been reported by [1]. The $1 D$ Stefan problem is given by:

$$
\frac{\partial}{\partial t} T(x, t)=\alpha \frac{\partial^{2}}{\partial x^{2}} T(x, t), 0 \leqslant x \leqslant x_{\Gamma}(t)
$$

with boundary conditions, $T(0, t)=T_{w}, T(x, t)=T_{s a t}, x \geqslant x_{\Gamma}(t), \rho_{v} h_{l v} \frac{\partial}{\partial t} x_{\Gamma}(t)=-\lambda_{v} \frac{\partial}{\partial x} T\left(x_{\Gamma}, t\right)$. The an- 
(a)

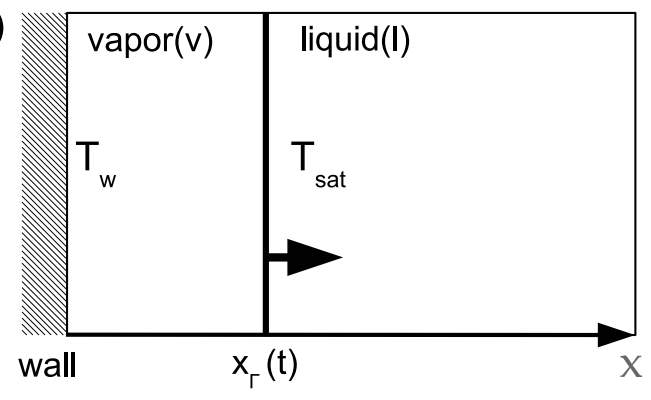

(b)

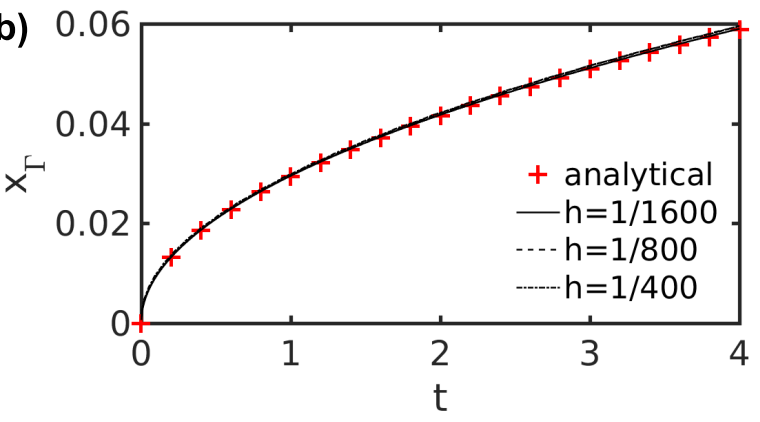

Figure 1: (a) Computational set-up for the 1D Stefan problem, (b) Comparison of numerical results obtained by the unstructured CLS method with phase change, and analytical solution [1].

alytical solution for $x_{\Gamma}(t)[1]$ is written as:

$$
x_{\Gamma}(t)=2 \zeta \sqrt{\alpha_{v} t}
$$

where $\zeta$ is the solution of the transcendental equation $\zeta \exp \left(\zeta^{2}\right) \operatorname{erf}(\zeta)=\frac{c_{p, v}\left(T_{w}-T_{s a t}\right)}{\sqrt{\pi} h_{v v}}$, and $\alpha_{v}$ is defined as the thermal diffusivity of the vapor phase. Comparison of numerical and analytical solutions is illustrated in Fig. 1b. Numerical results converge to the analytical solution upon grid refinement, which demonstrates that numerical methods for liquid-vapor phase change have been well implemented.

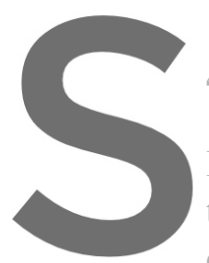

4.2 Filim boiling on a horizontal cylinder

In this section, film boiling phenomenon or a

trated in Fig. 2. Neunann boundary conditions

condition for the velocity and $T\left(\mathbf{x}_{w}, t\right)=T_{w}$ on th
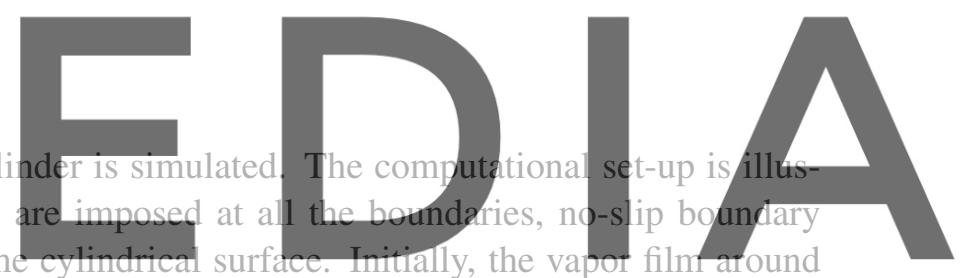

the cylinder with diameter $D$ is uniformly distributed with a thickness of $0.1 D$, whereas a liquid-vapor

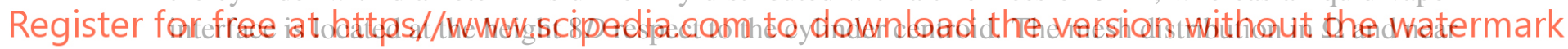

the cylinder where mesh resolution is maximized, is shown in Fig. 2. The grid size around the cylinder is $h \approx D / 200$, and the computational domain is divided in 52936 triangular prisms. As initial conditions, the velocity field is zero, and temperature field in $\Omega$ is equal to the saturation temperature $T_{\text {sat }}$.

Film boiling on a cylinder can be characterized by the Grashof number $(G r)$, Prandtl number $\left(P r_{v}\right)$, Jakob number $\left(J a_{v}\right)$, and physical properties ratios, which are defined as follows:

$$
\mathrm{Gr}=\frac{\rho_{v}\left(\rho_{l}-\rho_{v}\right) \| \mathbf{g}|| D^{3}}{\mu_{v}^{2}}, \operatorname{Pr}_{v}=\frac{\mu_{v} c_{p, v}}{\lambda_{v}}, \mathrm{Ja}_{v}=\frac{c_{p, v}\left(T_{w}-T_{s a t}\right)}{h_{l v}}, \frac{\rho_{v}}{\rho_{l}}, \frac{\mu_{v}}{\mu_{l}}, \frac{c_{p, v}}{c_{p, l}}, \frac{\lambda_{v}}{\lambda_{l}} .
$$

In this research, $\mathrm{Ja}_{v}=0.1, \operatorname{Pr}_{v}=4.22, \mathrm{Gr}=\{1000,4000,8000,10000\}, \rho_{v} / \rho_{l}=\mu_{v} / \mu_{l}=\lambda_{v} / \lambda_{l}=$ $c_{p, v} / c_{p, l}=0.01$. The most dangerous wavelength $\left(l_{d}\right)$ [17] of Rayleigh-Taylor instability is defined as $l_{d}=2 \pi\left(\left(3 \sigma /\left(\left(\rho_{l}-\rho_{v}\right)\|\mathbf{g}\|\right)\right)^{1 / 2}=8 D\right.$. Characteristic length $\left(l_{s}\right)$, characteristic velocity $\left(v_{s}\right)$ and characteristic time $\left(t_{s}\right)$ scales are defined as $l_{s}=D, v_{s}=\left(\|\mathbf{g}\| l_{s}\right)^{1 / 2}$, and $t_{s}=l_{s} / v_{s}$. Finally, the Nusselt number $(\mathrm{Nu})$ is computed as follows:

$$
\mathrm{Nu}\left(\mathbf{x}_{w}, t\right)=\frac{D}{\left(T_{w}-T_{\text {sat }}\right)}\left(\nabla T \cdot \mathbf{e}_{w}\right)\left(\mathbf{x}_{w}, t\right), \overline{\mathrm{Nu}}(t)=\frac{1}{A_{w}} \int_{A_{w}} \mathrm{Nu}\left(\mathbf{x}_{w}, t\right) d A, \overline{\overline{\mathrm{Nu}}}=\frac{1}{T} \int_{0}^{T} \overline{\mathrm{Nu}}(t) d t,
$$



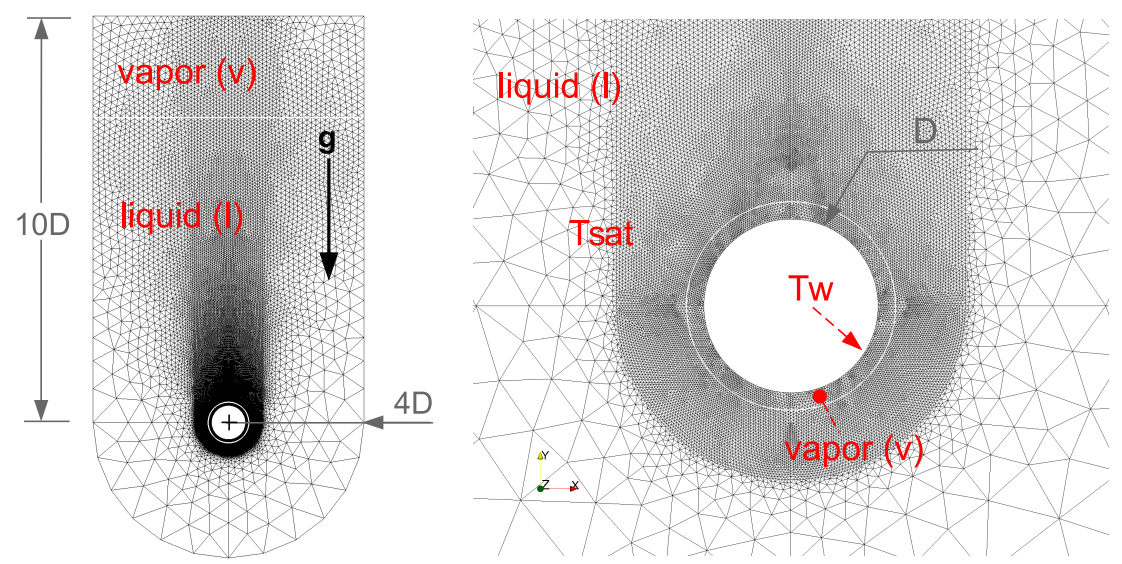

Figure 2: Computational set-up and mesh distribution for simulation of film boiling on a cylinder.

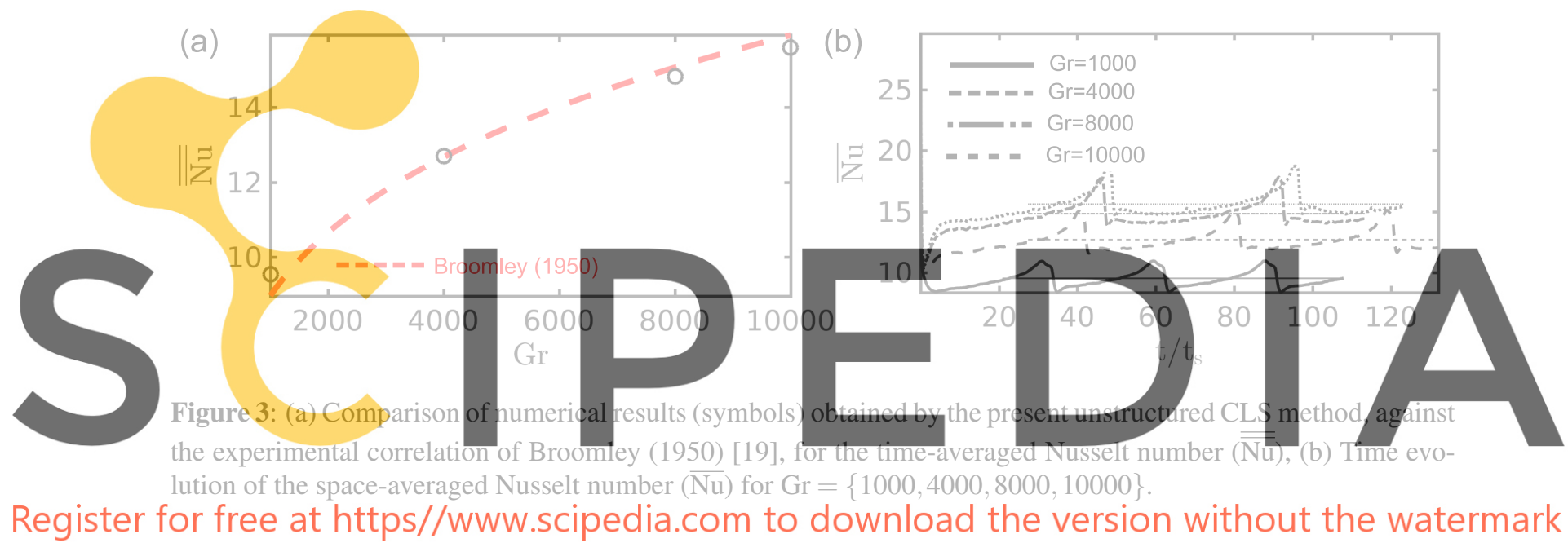

where $\mathrm{Nu}\left(\mathbf{x}_{w}, t\right)$ is the local Nusselt number, $\overline{\mathrm{Nu}}(t)$ is the space-averaged Nusselt number at the time $t$,

$A_{w}$ is the wall surface, and $\overline{\mathrm{Nu}}$ is the time-averaged Nusselt number in the period $T$.

Using boundary-layer approximations, [19] predicted the Nusselt number for film boiling on a cylinder as follows, $\mathrm{Nu}_{B}=0.62\left(\mathrm{Gr}_{\operatorname{Pr}} /\left(1+0.34 \mathrm{Ja}_{v}\right)\right)^{1 / 4}$, where the constant was adjusted from experimental data. Fig.3a shows that the Nusselt number computed by the the novel unstructured CLS method is in close agreement with $\mathrm{Nu}_{B}$ [19]. Fig.3b depicts the time evolution of Nusselt number $(\overline{N u})$ for $1000 \leq$ $\mathrm{Gr} \leq 10000$. Finally, Fig. 4 shows the evolution of the fluid interface. In the beginning a vapor film covers the cylinder, and then bubbles release from the upper region, driven by Taylor instability.

\section{CONCLUSIONS}

- An unstructured CLS method for two-phase flow with liquid-vapor phase change has been introduced by the first time. The numerical model has been verified and validated against analytical solutions and experimental correlations from the literature. The unstructured CLS method is 

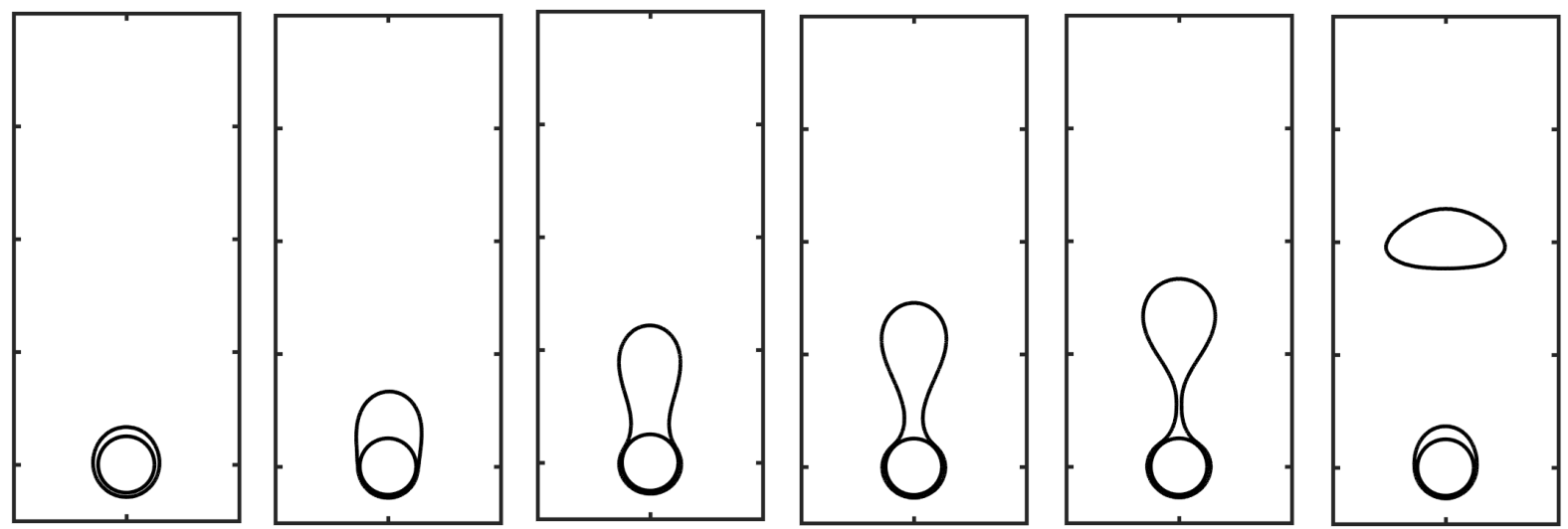

Figure 4: Time evolution of interface in film boiling $\left(\mathrm{Ja}_{v}=0.1, \operatorname{Pr}_{v}=4.22, \mathrm{Gr}=10000\right)$ on a cylinder for $t / t_{S}=\{1.1,22.4,44.9,47.1,48.3,50.5\}$

second-order accurate.

- Unstructured flux-limiters schemes proposed by $[10,15]$ to discretize convective term of transport equations, improves the numerical stability of the liquid-vapor phase change solver.

Altogether, numerical schemes have lead to a robust and accurate numerical model for simulation of liquid-vapor phase change on unstructured meshes.

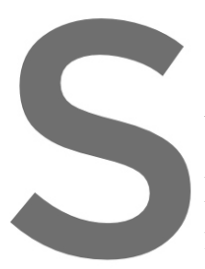

ACKNOWLED GMIENTS
N. Balcázar-Arciniega, as
for the financial suppoit th
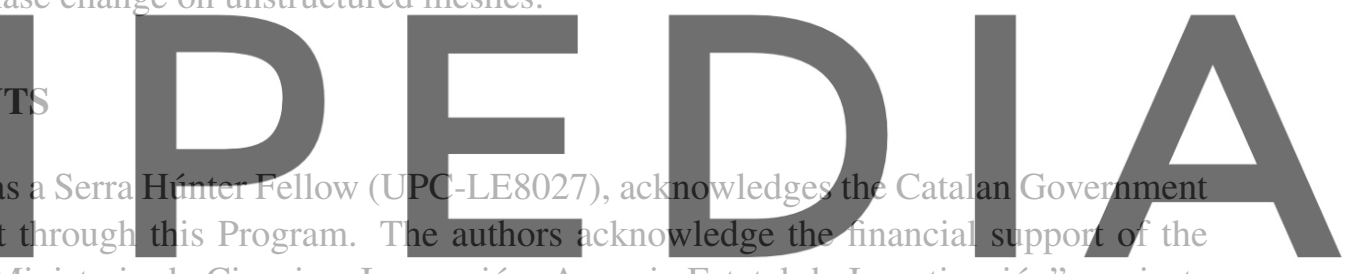

Spanish Government, "Ministerio de Ciencia e Innovación, Agencia Estatal de Investigación", projects

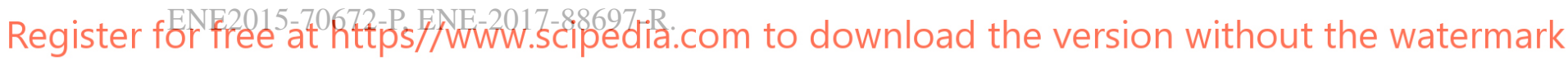

\section{REFERENCES}

[1] Alexiades, V., And Solomon, A. D. Mathematical Modeling of Melting and Freezing Processes, 1st ed. CRC Press, 1993.

[2] Amani, A., Balcázar, N., Castro, J., And Oliva, A. Numerical study of droplet deformation in shear flow using a conservative level-set method. Chemical Engineering Science 207 (2019), 153-171.

[3] Amani, A., Balcázar, N., NAseri, A., And Rigola, J. A numerical approach for nonNewtonian two-phase flows using a conservative level-set method. Chemical Engineering Journal 385 (2020), 123896.

[4] Antepara, O., Balcázar, N., And Oliva, A. Tetrahedral adaptive mesh refinement for twophase flows using conservative levelset method. International Journal for Numerical Methods in Fluids 93, 2 (2021), 481-503. 
[5] Antepara, O., Balcázar, N., Rigola, J., And Oliva, A. Numerical study of rising bubbles with path instability using conservative level-set and adaptive mesh refinement. Computers and Fluids 187 (2019), 83-97.

[6] Balcázar, N., Antepara, O., Rigola, J., And Oliva, A. DNS of Thermocapillary Migration of Deformable Droplets. In ERCOFTAC Series, vol. 25. 2019, pp. 207-213.

[7] Balcázar, N., Antepara, O., Rigola, J., And Oliva, A. DNS of Drag-Force and Reactive Mass Transfer in Gravity-Driven Bubbly Flows. In ERCOFTAC Series. 2020.

[8] Balcázar, N., Castro, J., Chiva, J., And Oliva, A. DNS of falling droplets in a vertical channel. International Journal of Computational Methods and Experimental Measurements 6, 2 (2017), 398-410.

[9] Balcázar, N., Castro, J., Rigola, J., AND Oliva, A. DNS of the wall effect on the motion of bubble swarms. Procedia Computer Science 108 (2017), 2008-2017.

[10] Balcázar, N., Jofre, L., Lehmkuhl, O., Castro, J., And Rigola, J. A finitevolume/level-set method for simulating two-phase flows on unstructured grids. International Journal of Multiphase Flow 64 (2014), 55-72.

[11] BAlć́zar, N., Lehmkuhl, O., Jofre, L., And Oliva, A. Level-set simulations of buoyancydriven motion of single and multiple bubbles. International Journal of Heat and Fluid Flow 56 (2015).
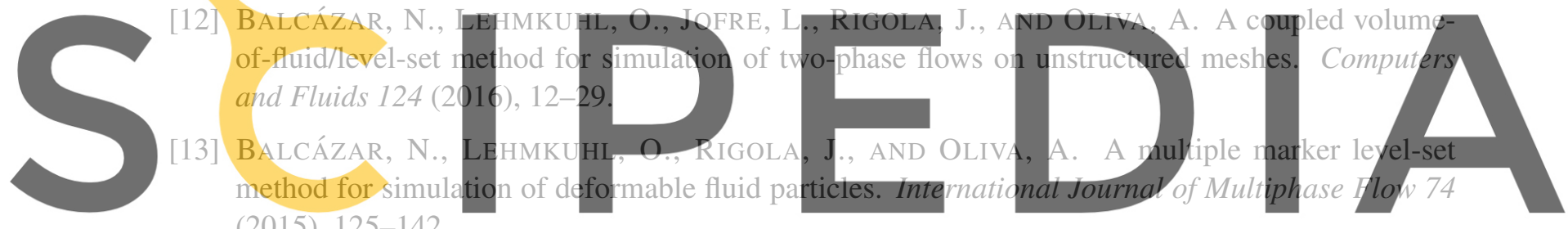
(2015), 125-142.

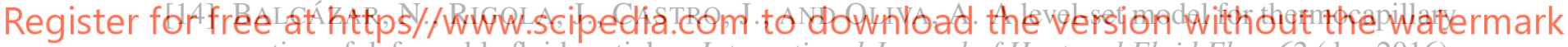
motion of deformable fluid particles. International Journal of Heat and Fluid Flow 62 (dec 2016), 324-343.

[15] Balcázar-Arciniega, N., Antepara, O., Rigola, J., And Oliva, A. A level-set model for mass transfer in bubbly flows. International Journal of Heat and Mass Transfer 138 (2019), $335-356$.

[16] Balcázar-Arciniega, N., Rigola, J., And Oliva, A. DNS of Mass Transfer from Bubbles Rising in a Vertical Channel. In Lecture Notes in Computer Science (including subseries Lecture Notes in Artificial Intelligence and Lecture Notes in Bioinformatics), vol. 11539 LNCS. 2019, pp. 596-610.

[17] Berenson, P. J. Film-Boiling Heat Transfer From a Horizontal Surface. Journal of Heat Transfer 83, 3 (1961), 351-356.

[18] Brackbill, J., Kothe, D., And Zemach, C. A continuum method for modeling surface tension. Journal of Computational Physics 100, 2 (1992), 335-354. 
[19] Bromley, L. A. Heat transfer in stable film boiling. Chemical Engineering Progress (1950).

[20] ChORIN, A. J. Numerical Solution of the Navier-Stokes Equations. Mathematics of Computation 22, 104 (1968), 745.

[21] Esmaeeli, A., And Tryggvason, G. A front tracking method for computations of boiling in complex geometries. International Journal of Multiphase Flow 30, 7-8 (jul 2004), 1037-1050.

[22] Esmaeeli, A., And Tryggvason, G. Computations of film boiling. Part I: numerical method. International Journal of Heat and Mass Transfer 47, 25 (dec 2004), 5451-5461.

[23] Gibou, F., Chen, L., Nguyen, D., and Banerjee, S. A level set based sharp interface method for the multiphase incompressible NavierStokes equations with phase change. Journal of Computational Physics 222, 2 (mar 2007), 536-555.

[24] Gibou, F., Fedkiw, R., ANd Osher, S. A review of level-set methods and some recent applications. Journal of Computational Physics 353 (jan 2018), 82-109.

[25] Gibou, F., Fediw, R. P., Cheng, L.-T., And Kang, M. A Second-Order-Accurate Symmetric Discretization of the Poisson Equation on Irregular Domains. Journal of Computational Physics 176, 1 (feb 2002), 205-227.

[26] GutiérRez, E., Balcázar, N., Bartrons, E., And Rigola, J. Numerical study of Taylor bubbles rising in a stagnant liquid using a level-set/moving-mesh method. Chemical Engineering Science 164 (2017), 158-177.
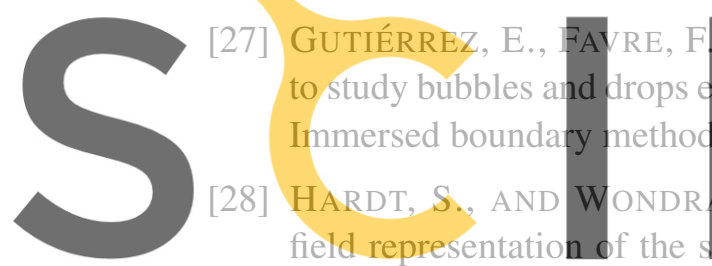
field representation of $5871-5895$

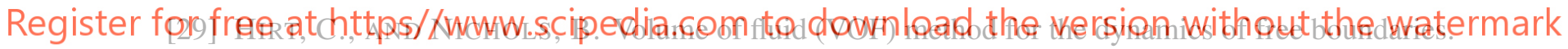
Journal of Computational Physics 39, 1 (jan 1981), 201-225.

[30] IRfan, M., AND Muradoglu, M. A front tracking method for direct numerical simulation of evaporation process in a multiphase system. Journal of Computational Physics 337 (may 2017), $132-153$.

[31] Juric, D., And Tryggvason, G. Computations of boiling flows. International Journal of Multiphase Flow 24, 3 (apr 1998), 387-410.

[32] Karniadakis, G. E., And Kirby II, R. M. Parallel Scientific Computing in C++ and MPI. Cambridge University Press, jun 2003.

[33] Kumar Singh, N., And Premachandran, B. A coupled level set and volume of fluid method on unstructured grids for the direct numerical simulations of two-phase flows including phase change. International Journal of Heat and Mass Transfer 122 (jul 2018), 182-203.

[34] MA, C., AND Bothe, D. Numerical modeling of thermocapillary two-phase flows with evaporation using a two-scalar approach for heat transfer. Journal of Computational Physics 233 (jan 
2013), 552-573.

[35] Ningegowda, B., Ge, Z., Lupo, G., Brandt, L., And Duwig, C. A mass-preserving interface-correction level set/ghost fluid method for modeling of three-dimensional boiling flows. International Journal of Heat and Mass Transfer 162 (dec 2020), 120382.

[36] Ningegowda, B., And Premachandran, B. A Coupled Level Set and Volume of Fluid method with multi-directional advection algorithms for two-phase flows with and without phase change. International Journal of Heat and Mass Transfer 79 (dec 2014), 532-550.

[37] Olsson, E., AND Kreiss, G. A conservative level set method for two phase flow. Journal of Computational Physics 210, 1 (nov 2005), 225-246.

[38] OSher, S., AND Sethian, J. A. Fronts propagating with curvature-dependent speed: Algorithms based on Hamilton-Jacobi formulations. Journal of Computational Physics 79, 1 (nov 1988), 12 49.

[39] Perez-Raya, I., And Kandlikar, S. G. Discretization and implementation of a sharp interface model for interfacial heat and mass transfer during bubble growth. International Journal of Heat and Mass Transfer 116 (jan 2018), 30-49.

[40] Prosperetti, A., And Tryggvason, G. Computational Methods for Multiphase Flow. Cambridge University Press, Cambridge, 2007.

[41] Rajkotwala, A., Panda, A., Peters, E., Baltussen, M., Van der Geld, C., Kuerten, J., AND KUIPERS, J. A critical comparison of smooth and sharp interface methods for phase transition. International Journal of Multiphase Flow 120 (nov 2019), 103093.

[42] Rhie, C. M., AND ChOw, W. L. Numerical study of the turbulent flow past an airfoil with trailing edge separation. AIAA Journal 21, 11 (1983), 1525-1532.

[43] Rider, W. J., And Kothe, D. B. Reconstructing Volume Tracking. Journal of Computational Physics 141, 2 (apr 1998), 112-152.

[44] SATO, Y., AND NiČENO, B. A sharp-interface phase change model for a mass-conservative interface tracking method. Journal of Computational Physics 249 (sep 2013), 127-161.

[45] Schillaci, E., Antepara, O., Balcázar, N., Serrano, J. R., And Oliva, A. A numerical study of liquid atomization regimes by means of conservative level-set simulations. Computers and Fluids 179 (2019), 137-149.

[46] Son, G., AND DHIR, V. K. Numerical Simulation of Saturated Film Boiling on a Horizontal Surface. Journal of Heat Transfer 119, 3 (aug 1997), 525-533.

[47] Son, G., AND DhIR, V. K. Numerical Simulation of Film Boiling Near Critical Pressures With a Level Set Method, feb 1998.

[48] Son, G., AND DHIR, V. K. Numerical simulation of nucleate boiling on a horizontal surface at high heat fluxes. International Journal of Heat and Mass Transfer 51, 9-10 (may 2008), 2566-2582.

[49] Son, G., AND DHIR, V. K. Three-dimensional simulation of saturated film boiling on a horizontal cylinder. International Journal of Heat and Mass Transfer 51, 5-6 (mar 2008), 1156-1167.

[50] Son, G., Dhir, V. K., And Ramanujapu, N. Dynamics and Heat Transfer Associated With a 
Single Bubble During Nucleate Boiling on a Horizontal Surface. Journal of Heat Transfer 121, 3 (aug 1999), 623-631.

[51] SUn, D., AND TAO, W. A coupled volume-of-fluid and level set (VOSET) method for computing incompressible two-phase flows. International Journal of Heat and Mass Transfer 53, 4 (jan 2010), $645-655$.

[52] Sussman, M., And Puckett, E. G. A Coupled Level Set and Volume-of-Fluid Method for Computing 3D and Axisymmetric Incompressible Two-Phase Flows. Journal of Computational Physics 162, 2 (aug 2000), 301-337.

[53] Sussman, M., Smereka, P., ANd Osher, S. A Level Set Approach for Computing Solutions to Incompressible Two-Phase Flow. Journal of Computational Physics 114, 1 (sep 1994), 146-159.

[54] Tanguy, S., Ménard, T., And Berlemont, A. A Level Set Method for vaporizing two-phase flows. Journal of Computational Physics 221, 2 (feb 2007), 837-853.

[55] Termo Fluids S.L. http://www.termofluids.com/, 2020.

[56] Tomar, G., Biswas, G., Sharma, A., And Welch, S. W. J. Multimode analysis of bubble growth in saturated film boiling. Physics of Fluids 20, 9 (sep 2008), 092101.

[57] Tryggvason, G., Bunner, B., Esmaeeli, A., Juric, D., Al-Rawahi, N., Tauber, W., HAN, J., NAS, S., AND JAN, Y.-J. A Front-Tracking Method for the Computations of Multiphase Flow. Journal of Computational Physics 169, 2 (may 2001), 708-759.

[58] Tryggvason, G., Bunner, B., Esmaeeli, A., Juric, D., Al-Rawahi, N., Tauber, W., HAN, J., NAS, S., AND JAN, Y.-J. A Front-Tracking Method for the Computations of Multiphase Flow. Journal of Computational Physics 169, 2 (may 2001), 708-759.

[59] Tryggvason, G., Esmaeeli, A., And Al-Rawahi, N. Direct numerical simulations of flows with phase change. Computers and Structures 83, 6-7 (feb 2005), 445-453.

[60] Tryggvason, G., SCARdovelli, R., And Zaleski, S. The volume-of-fluid method. In Direct Numerical Simulations of GasLiquid Multiphase Flows. Cambridge University Press, jan 2001, pp. 95-132.

[61] Tryggvason, G., Scardovelli, R., AND Zaleski, S. Direct numerical simulations of GasLiquid multiphase flows. 2011.

[62] TsUI, Y.-Y., AND LIN, S.-W. Three-dimensional modeling of fluid dynamics and heat transfer for two-fluid or phase change flows. International Journal of Heat and Mass Transfer 93 (feb 2016), 337-348.

[63] Welch, S. W., AND Wilson, J. A Volume of Fluid Based Method for Fluid Flows with Phase Change. Journal of Computational Physics 160, 2 (may 2000), 662-682.

[64] YUAN, M., YANG, Y., LI, T., AND HU, Z. Numerical simulation of film boiling on a sphere with a volume of fluid interface tracking method. International Journal of Heat and Mass Transfer 51, 7-8 (apr 2008), 1646-1657. 